\title{
Comparison of chromoendoscopy and conventional endoscopy in the detection of premalignant gastric lesions
}

\author{
Seyed Alireza Taghavi MD¹, Mohammed Ebrahim Membari MD², Ahad Eshraghian MD ${ }^{1,2}$, \\ Seyed Moshen Dehghani MD', Lelah Hamidpour MD¹, Farnaz Khademalhoseini MD
}

SA Taghavi, ME Membari, A Eshraghian, SM Dehghani, L Hamidpour, F Khademalhoseini. Comparison of chromoendoscopy and conventional endoscopy in the detection of premalignant gastric lesions. Can J Gastroenterol 2009;23(2):105-108.

BACKGROUND: Diagnosis and localization of intestinal metaplasia and early gastric cancer is problematic because of the lack of any telltale gross endoscopic signs.

OBJECTIVE: To compare the efficacy of chromoendoscopy with conventional endoscopy for the detection of gastric premalignant lesions (intestinal metaplasia).

METHOD: Thirty-three patients in whom previous routine endoscopic biopsies showed intestinal metaplasia were enrolled in a prospective study. Each patient underwent a two-step endoscopy procedure: conventional endoscopy and chromoendoscopy using methylene blue. Biopsies were taken during each step and were studied by an expert pathologist. Presence of intestinal metaplasia was considered a positive result.

RESULTS: Considering the presence of intestinal metaplasia anywhere in the stomach as a positive result, 13 patients were diagnosed with intestinal metaplasia using both endoscopic methods, while eight patients had positive results using chromoendoscopy without any metaplastic changes detected with the conventional method $(\mathrm{P}=0.003)$. One patient showed positive biopsies with the conventional method while the pathology report showed no positive biopsies using the chromoendoscopy method. The number of positive biopsies from the antrum, body and fundus were 18, 15 and seven, respectively, using chromoendoscopy, and 10, four and two, respectively, from the same sites using conventional endoscopy.

CONCLUSION: The present study demonstrated that chromoendoscopy is superior to conventional endoscopy for the detection of metaplastic changes and its use can be suggested for the screening of early malignancies of the stomach.

Key Words: Chromoendoscopy; Conventional endoscopy; Intestinal metaplasia; Methylene blue; Mucosal biopsy

astric cancer is one of the most common malignant dis-

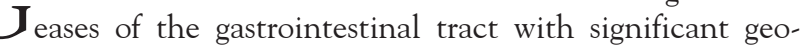
graphical, ethnic and socioeconomic variations in distribution (1). Substantial interest has arisen in recent years in the diagnosis and management of early gastric cancer and premalignant lesions of the gastric mucosa because of the high cure rate achieved with treatment of these lesions, compared with the poor prognosis of full-blown cancer (2-5). The generally accepted pathological sequence that leads to gastric adenocarcinoma is superficial gastritis followed by atrophic gastritis,

\section{Comparaison entre chromoendoscopie et endoscopie classique dans le dépistage des lésions gastriques prénéoplasiques}

\begin{abstract}
HISTORIQUE : Le diagnostic et la localisation de la métaplasie intestinale et du cancer superficiel de l'estomac posent problème en raison de l'absence de signes endoscopiques macroscopiques francs.

OBJECTIFS : Comparer l'efficacité de la chromoendoscopie à celle de l'endoscopie classique pour le dépistage des lésions gastriques prénéoplasiques (métaplasie intestinale).

MÉTHODES : Trente-trois patients chez qui des biopsies endoscopiques de routine avaient déjà révélé une métaplasie intestinale ont été inscrits à une étude prospective. Chaque patient a subi une endoscopie en deux étapes, c'est-à-dire, endoscopie classique suivie de chromoendoscopie au bleu de méthylène. Des biopsies ont été prélevées à chaque étape et ont été analysées par un anatomopathologiste. La présence de métaplasie intestinale était considérée comme un résultat positif.

RÉSULTATS : La présence de métaplasie intestinale, où que ce soit dans l'estomac, étant considérée comme un résultat positif, 13 patients ont reçu un diagnostic de métaplasie intestinale à l'aide des deux méthodes endoscopiques, tandis que huit patients ont présenté des résultats positifs à la chromoendoscopie, sans anomalies métaplasiques décelées à l'endoscopie classique $(\mathrm{p}=0,003)$. Un patient a obtenu des biopsies positives avec la méthode classique, tandis que le rapport d'anatomopathologie ne faisait état d'aucune biopsie positive à la chromoendoscopie. Le nombre de biopsies positives au niveau de l'antre pylorique, du corps et du fond de l'estomac a été respectivement de 18, 15 et sept à la chromoendoscopie et de 10 , quatre et deux aux mêmes points à l'endoscopie classique.

CONCLUSION : Cette étude a démontré que la chromoendoscopie est plus efficace que l'endoscopie classique pour le dépistage des anomalies métaplasiques et son utilisation est suggérée pour reconnaître le cancer superficiel de l'estomac.
\end{abstract}

\footnotetext{
${ }^{1}$ Nemazee Hospital, Shiraz University of Medical Sciences; ${ }^{2}$ Bandar Abbass University of Medical Science, Bandar Abbas, Iran

Correspondence: Dr Ahad Eshraghian, Gastroenterohepatology Research Centre of Shiraz University of Medical Sciences, Jahrom University of

Medical Science, PO Box 71345-1744, Shiraz, Iran. Telephone 98-711-627-6212, fax 98-711-627-6212, e-mail eshraghiana@yahoo.com

Received for publication August 7, 2008. Accepted December 1, 2008
} 
early gastric cancer, dysplasia or incomplete intestinal metaplasia) with routine biopsies, finding the exact location of previously sampled sites for endoscopic or surgical treatment or resampling is extremely difficult (9).

Methylene blue staining was originally described by Ida et al (9) to improve the diagnosis of early gastric cancer. Methylene blue dye is taken up by actively absorbing tissues such as the small intestine or colon epithelia. It does not stain nonabsorptive epithelia such as stratified squamous epithelium of the esophagus or normal gastric mucosa (10).

Methylene blue has recently been used to highlight subtle mucosal changes in the small intestine and colon, and to positively stain metaplastic absorptive epithelia such as intestinal type metaplasia in the stomach (11).

Although it is generally believed that chromoendoscopy can increase the rate of diagnosis of early cancers and precancerous lesions by enhancing visualization, no study has directly compared the accuracy and sensitivity of this method with conventional endoscopy and systematic biopsies. It should also be noted that this method is a time-consuming and costly procedure compared with upper endoscopy and systematic random biopsies; therefore, reliable evidence to show its efficacy is necessary before it can be recommended for use.

Because the prevalence of gastric cancer is high in Saudi Arabia, and premalignant lesions of the gastric mucosa are not infrequently encountered in our practice (12), the problems of localization and biopsy of these lesions are daily challenges. The present study was designed to compare the accuracy and sensitivity of chromoendoscopy with conventional endoscopy and systematic biopsies for locating premalignant lesions of the gastric mucosa.

\section{Patients}

\section{METHODS}

The present prospective study was performed in the Department of Gastroenterology at the Shiraz University of Medical Sciences, Shiraz, Iran.

During a period of one year between September 2006 and September 2007, 84 patients were diagnosed with intestinal metaplasia. Of these, 33 patients in whom previous endoscopic biopsies had shown intestinal metaplasia were enrolled in the study.

Inclusion criteria were an age of 18 to 65 years and a biopsyconfirmed intestinal metaplasia in a recent (less than six months) upper gastrointestinal endoscopy.

Exclusion criteria were coagulopathy, pre-existing or newly diagnosed gastric cancer, grossly visible mucosal lesions in a previous endoscopy, recent upper gastrointestinal bleeding or surgery leading to anatomical changes of the stomach, and comorbid diseases that may affect tolerance to upper endoscopy.

Patients were informed of the possible risks and benefits of participation in the study and written consent was obtained from each patient before enrollment. The Shiraz University of Medical Science's ethics committee approved the study. Repeat endoscopy was not only used in the study, but also was part of each patient's routine follow-up for neoplasia.

Demographic data including sex and age, any history of previous gastric endoscopic lesions, previous pathology and/or surgery of stomach, family history of gastric cancer, drug history, and clinical gastrointestinal signs and symptoms were recorded.

Patients underwent the endoscopy procedure using the Olympus GF-Q160 endoscope (Olympus, Japan). After standard sedation with $5 \mathrm{mg}$ of intravenous midazolam, additional sedation was given if needed during the procedure. Continuous monitoring of pulse and oxygen saturation was performed during the procedure.

\section{Conventional endoscopy}

Each procedure was performed in two phases: the first phase was a conventional endoscopy with a meticulous search of the gastric mucosa for grossly visible abnormalities. Washing with tap water and the use of anti-foam agents was performed when needed. All grossly visible lesions were sampled first; however, if no gross lesion was found, six random biopsies were taken (two from the antrum, two from the body and two from the fundus) according to the Sydney protocol (13).

\section{Chromoendoscopy}

The second phase was performed during the same endoscopic session without withdrawing the endoscope. First, all biopsy sites were vigorously washed with tap water and residual blood or fluid was removed with suction. The surface to be examined was sprayed with $20 \mathrm{~mL}$ of $10 \% \mathrm{~N}$-acetyl cystein (Aurum Pharmaceuticals Ltd, UK) using a specialized spray catheter (Endoflex, GmbH Germany) and all air was suctioned afterward. The $\mathrm{N}$-acetyl cystein was allowed to remain in contact with the gastric surface for $3 \mathrm{~min}$. Thereafter, $30 \mathrm{~mL}$ of $5 \%$ methylene blue solution (Aguattant Ltd, UK) was sprayed onto the gastric mucosa and was allowed to remain in contact for $3 \mathrm{~min}$ followed by extensive irrigation with tap water. Multiple biopsies were taken from all blue-stained spots regardless of whether biopsies were taken from the same spot during the conventional upper endoscopy.

\section{Histopathology}

The mean elapsed time for the complete procedure was 20 $\mathrm{min}$. There were no complications related to the procedure. Biopsies were labelled with alphabetic codes only and referred to an expert pathologist blind to the patient's name or procedure type. Biopsies that showed intestinal metaplasia were considered to be positive. To control for the possible effect of biopsy artefacts on visualization during the first phase, and staining or biopsies at the second phase endoscopies, all procedures were supervised by a gastroenterologist who was not a coauthor of the present study and was blind to the study protocol.

\section{Statistical analysis}

Data were analyzed by two-tail Fisher's exact and $\chi^{2}$ tests using SPSS software version 12.0 (SPSS Inc, Japan). A value of $\mathrm{P}<0.05$ was considered to be statistically significant.

\section{RESULTS}

Of 33 patients, 22 were men and 11 were women, with a mean $( \pm$ SD) age of $49.73 \pm 13.10$ years. Chromoendoscopy yeilded 40 positive biopsies compared with 16 positive biopsies when the conventional endoscopy method was used. The number of positive biopsies from the antrum, body and fundus was 18,15 and seven, respectively, using chromoendoscopy, and 10, four and two positive biopsies, respectively, from the same sites using conventional endoscopy.

The results from chromoendoscopy and the conventional method were concordant in 24 patients (72.7\%) (13 patients had intestinal metaplasia detected by both methods and 11 had 


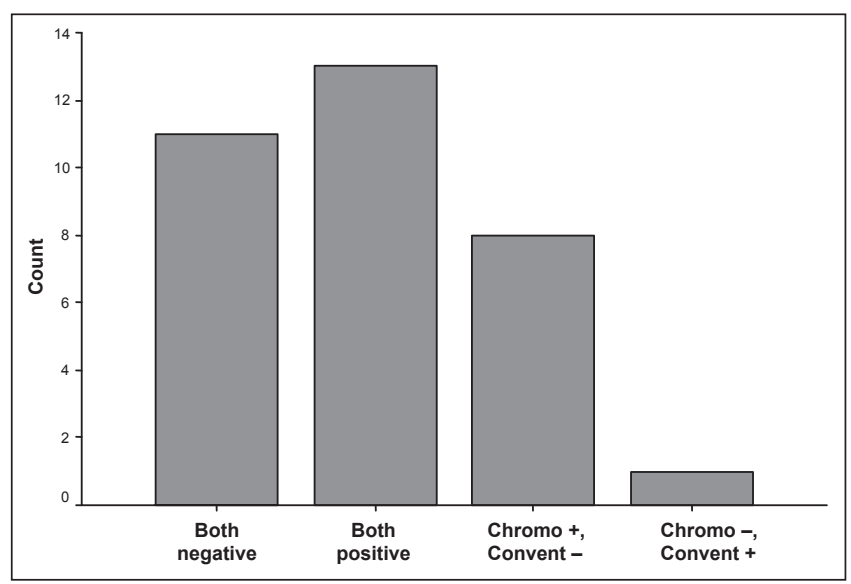

Figure 1) Concordance of conventional (Convent) endoscopy and chromoendoscopy (Chromo)

TABLE 1

Comparison of the number of involved anatomical segments detected with chromoendoscopy versus conventional endoscopy

\begin{tabular}{lcccc}
\hline & \multicolumn{4}{c}{ Anatomical segments involved, $\mathbf{n}$} \\
\cline { 2 - 5 } Endoscopy method & No involvement & Antrum & Body & Fundus \\
\hline Chromoendoscopy & 12 & 6 & 9 & 6 \\
Conventional endoscopy & 19 & 12 & 2 & 0 \\
\hline
\end{tabular}

negative results using both methods). In the remaining nine cases, the results from the two methods were discordant. Eight patients had positive results for intestinal metaplasia with chromoendoscopy while their pathology reports did not show any evidence of intestinal metaplasia using the conventional method. One patient had a positive biopsy detected by the conventional method while chromoendoscopy failed to detect any abnormality (Figure 1). The difference between chromoendoscopy-based biopsies versus conventional biopsies for the detection of intestinal metaplasia was statistically significant $(\mathrm{P}=0.003)$. The calculated sensitivity (considering all enrolled cases as established positive cases) was $63.6 \%$ for chromoendoscopy versus $42.4 \%$ for conventional endoscopy.

To assess the possible difference in efficacy of the two methods in the determination of the anatomical extent of histological abnormalities, the stomach was divided into three anatomical segments: the antrum, body and fundus. Chromoendoscopy was significantly more effective in demonstrating the involvement of additional anatomical segments ( $\mathrm{P}=0.002$ ) (Table 1).

Even in the group of patients with concordant positive results with both methods (13 patients), chromoendoscopy remained significantly more effective in demonstrating involvement of additional anatomical segments $(\mathrm{P}=0.01)$ (Table 2).

If superiority is defined as either the recognition of a histological abnormality in a patient with a negative result using the other method, or the identification of additional involved anatomical segments, chromoendoscopy was superior in 16 patients (48.5\%), while conventional endoscopy was superior in one patient $(3 \%)$, and both were equally effective in 16 patients (48.5\%) (Figure 2).

\section{DISCUSSION}

Detection of early cancers or precancerous lesions in the stomach may lead to better prognosis and survival (14). However,
TABLE 2

Comparison of the number of involved anatomical segments detected with chromoendoscopy versus conventional endoscopy in patients with concordant positive results using both methods

\begin{tabular}{lccc}
\hline \multirow{2}{*}{ Endoscopy method } & \multicolumn{3}{c}{ Anatomical segments involved, $\mathbf{n}$} \\
\cline { 2 - 4 } & Antrum & Body & Fundus \\
\hline Chromoendoscopy & 5 & 5 & 3 \\
Conventional endoscopy & 11 & 2 & 0 \\
\hline
\end{tabular}

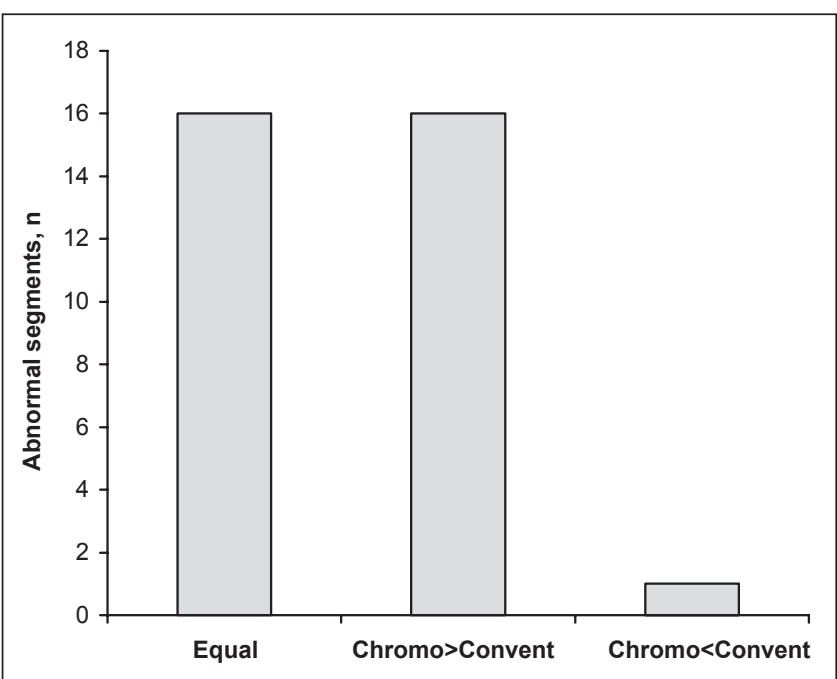

Figure 2) Comparison of the detection of abnormal segments by conventional (Convent) endoscopy and chromoendoscopy (Chromo)

establishment of an early detection or screening program is difficult, even in high-risk groups, because of the low validity and reliability of conventional endoscopic techniques for the detection of subtle histological changes. The same problems are encountered when determination of the extent of histological abnormalities is required to plan a surgical resection or endoscopic ablation therapy $(15,16)$.

Staining of the gastric mucosa with methylene blue to identify areas of nonacid-producing, mostly absorptive mucosa (ie, chromoendoscopy) has been used for a relatively long time $(10,12,13,17,18)$.

Chromoendoscopy is relatively simple and uses normal endoscopic equipment. However, its superiority over conventional endoscopy is rarely or never subjected to trial. The present study clearly demonstrated that in comparison with conventional endoscopy, chromoendoscopy is a superior method for the detection of at least one of the premalignant gastric lesions (intestinal metaplasia). We showed that staining with methylene blue is helpful for the accurate delineation of the anatomical extent of histological abnormalities in the stomach. This ability may be particularly helpful when considering recent trends in the nonsurgical (ie, endoscopic) treatment of these lesions. The accurate mapping of abnormal areas is of the utmost importance to allow for confident and reliable treatments when using noninvasive methods.

Based on the results of the present study, chromoendoscopy using methylene blue is an effective method for the detection and accurate delineation of at least some of the precancerous lesions of the stomach. Chromoendoscopy can be recommended when a follow-up endoscopy for confirmation of an initial abnormal result or mapping of abnormal area(s) is planned. 
Although no case of dysplasia or cancer was encountered in the present study (which may limit the application of the results to these more serious lesions), the superiority of chromoendoscopy for the detection of one of the premalignant gastric lesions (ie, intestinal metaplasia) is promising.

It should be noted that there may be a slight risk of bias toward chromoendoscopy because during chromoendoscopy, the endoscopist was already aware of the results of the conventional endoscopy. However, this risk should be minimal considering that biopsies during the chromoendoscopy phase were strictly limited to the stained areas and all procedures were supervised by an independent observer.

Because only patients with previous positive biopsy results were included, the specificity of these procedures cannot be calculated based on data from the current study. The calculated sensitivity should also be regarded as an approximation, and

\section{REFERENCES}

1. Lawrence M. Early gastric cancer, twenty-eight-year experience. Ann Surg 1991;213:332-4.

2. Meyers WC, Damiano RJ Jr, Rotolo FS, Postlethwait RW. Adenocarcinoma of the stomach-changing patterns over the last 4 decades. Ann Surg 1987;205:1-8.

3. Korenaga D, Tsujitani S, Haraguchi M. Long-term survival in Japanese patients with far advanced carcinoma of the stomach. World J Surg 1988;12:236-40.

4. Mcbride CM, Boddie AW Jr. Adenocarcinoma of the stomach - are we making any progress? South Med J 1987;80:283-6.

5. Sakita T, Ogura Y, Takasu S. The development of endoscopic diagnosis of early carcinoma of the stomach. Jpn J Clin Oncol 1971;1:113-28.

6. Murakami T. Pathomorphological diagnosis. Definition and gross classification of early gastric cancer. Gann Monogr Cancer Res 1971;11:53.

7. Fennerty MB, Sampliner RE, McGee DL, Hixson LJ, Garewal HS. Intestinal metaplasia of the stomach: Identification by a selective mucosal staining technique. Gastrointest Endosc 1992;38:696-8.

8. Bralow SP. Early gastric cancer. Up to date 2005;13:2.

9. Ida K, Hashimoto Y, Takeda S, Murakami K, Kawai K. Endoscopic diagnosis of gastric cancer with dye scattering. Am J Gastroenterol 1975;63:316-20.

10. Niveloni S, Fiorini A, Dezi R, et al. Usefulness of videoduodenoscopy and vital dye staining as indicators of mucosal further studies including consecutive patients without a previous diagnosis of metaplastic lesion are needed for the above mentioned purposes.

Further studies with a larger number of patients with more diverse risks for other gastric histological abnormalities, ideally including a wider spectrum of histological changes, are needed to completely elucidate the role of chromoendoscopy in screening or follow-up of patients after surgical or endoscopic treatment.

ACKNOWLEDGEMENTS: The authors would like to express their gratitude to the nursing staff of the Faghihi Hospital endoscopy unit, Shiraz, Iran. This study was sponsored by the research council of the Shiraz University of Medical Sciences. There is no conflict of interest to declare.

atrophy of celiac disease: Assessment of interobserver agreement. Gastrointest Endosc 1998;47:223-9.

11. Fennery MB, Sampliner RE, McGee DL, et al. Intestinal metaplasia of the stomach: Identification by a selective mucosal staining technique. Gastrointest Endosc 1992;38:696-8.

12. Abdi-Rad A, Gaderi-Sohi S, Nadimi-Barfroosh H, Emami S. Trend in incidence of gastric adenocarcinoma by tumor location from 1969-2004: A study in one referral center in Iran. Diagn Pathol 2006;1:5.

13. Yoshida S. Endoscopic diagnosis and treatment of cancer in the alimentary tract. Digestion 1998;59:502-8.

14. Denis-Ribeiro M. Chromoendoscopy for early diagnosis of gastric cancer. Eur J Gastroenterol Hepatol 2006;18:831-8.

15. Dinis-Ribeiro M, Lopes C, da Costa-Pereira A, et al. A follow up model for patients with atrophic chronic gastritis and intestinal metaplasia. J Clin Pathol 2004;57:177-82.

16. Pasechnikov VD, Chukov SZ, Kotelevets SM, et al. Possibility of noninvasive diagnosis of gastric mucosal precancerous changes. World J Gastroenterol 2004;1:10-21.

17. Kida M, Kobayashi K, Siagenji K. Routine chromoendoscopy for gastrointestinal disease: Indications revised. Endoscopy 2003;35:590-6.

18. Shaw D, Blair V, Framp A, et al. Chromoendoscopic surveillance in hereditary diffuse gastric cancer: An alternative to prophylactic gastrectomy? Gut 2005;54:461-8. 


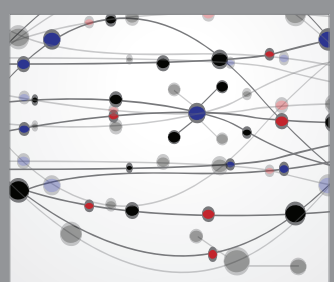

The Scientific World Journal
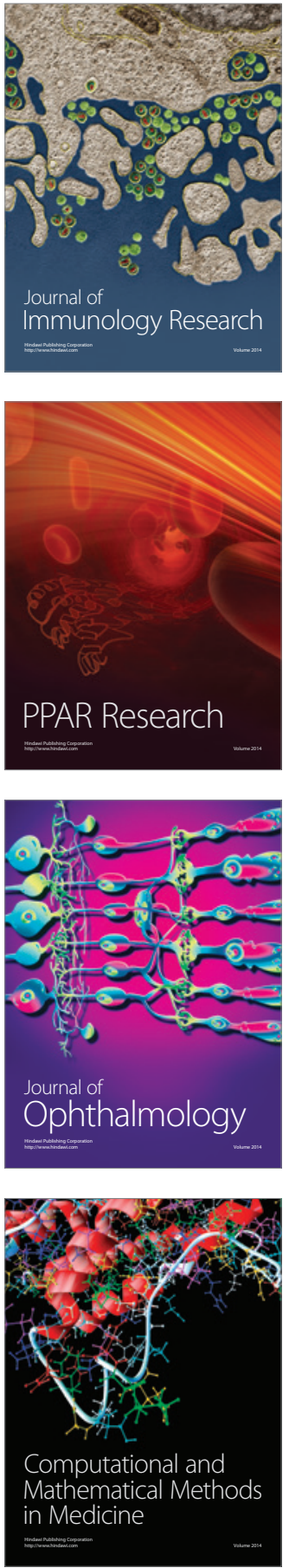

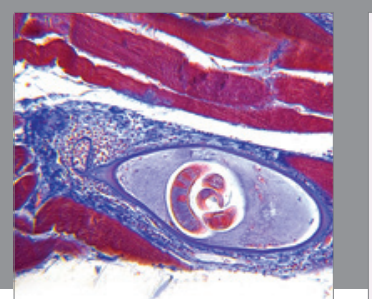

Gastroenterology Research and Practice

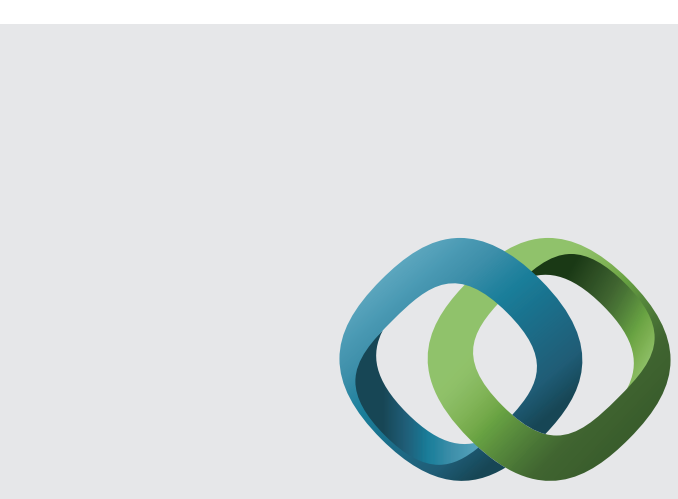

\section{Hindawi}

Submit your manuscripts at

http://www.hindawi.com
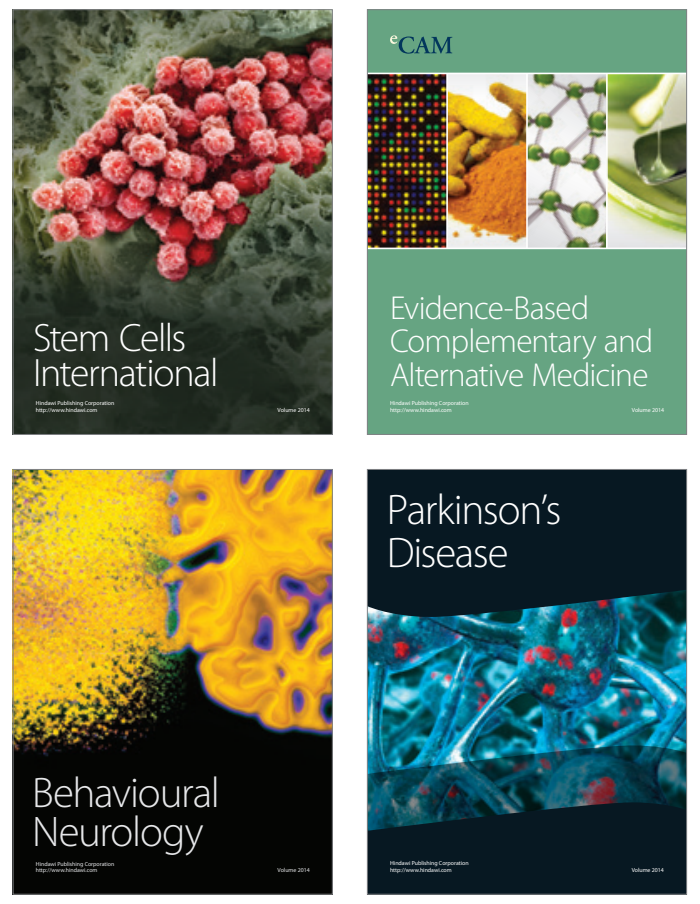
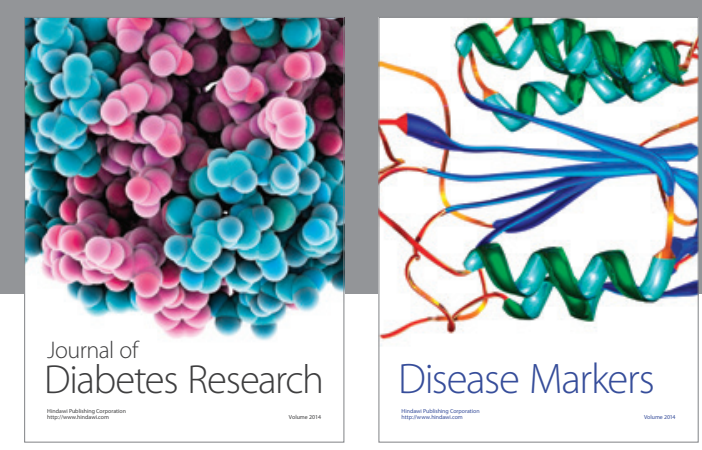

Disease Markers
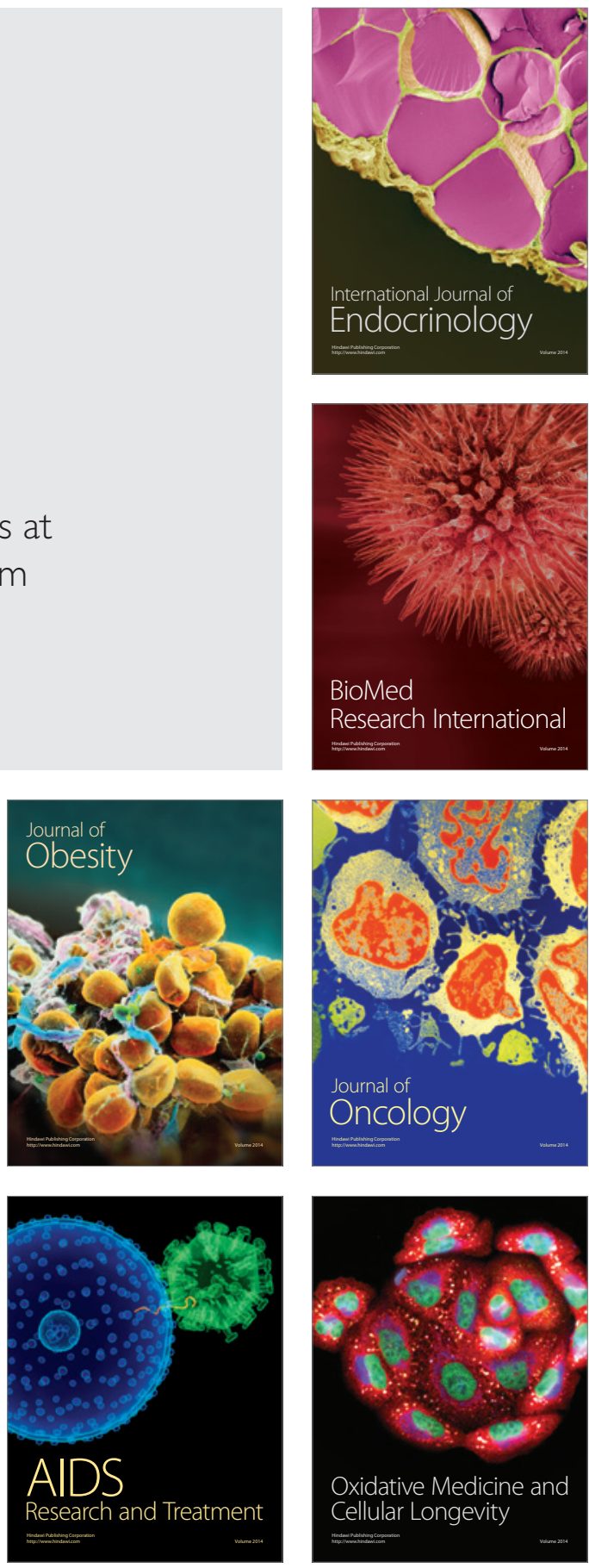